\title{
Cystic fibrosis mortality and survival in the
}

UK: 1947-2003

\author{
J.A. Dodge*, P.A. Lewis ${ }^{\#}$, M. Stanton" and J. Wilsher"
}

ABSTRACT: Data up to 1995 on the survival of 3-yr cohorts of patients with cystic fibrosis (CF) born in the UK in the period 1968-1992 have previously been published. The present study reports survival data up to the end of 2003 together with a 2003 population estimate.

All subjects with CF born in the UK in the period 1968-1992 were identified up to 1997 by active enquiry through recognised CF clinics and other hospital consultants. Information from the death certification authorities up to the end of 2003 was added. Death certificates that could not be matched with UK Cystic Fibrosis Survey records were investigated and the data reconciled.

The observed survival up to 2003 of CF patients born in 1978 was $55 \%$ for males and $49 \%$ for females. For 1988 and 1992 the data were 91 and $88 \%$, and 97 and $96 \%$, respectively. The estimated 2003 mid-year CF population was 8,284.

The continuing improvement in survival of cystic fibrosis patients in successive cohorts means that the previous prediction of median survival of $>50 \mathrm{yrs}$ of age for individuals born in 2000 continues to look realistic, even in the absence of proven effective therapy aimed at correcting the basic cystic fibrosis defect.

KEYWORDS: Cystic fibrosis, epidemiology, expectation of life, survival

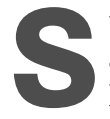
tandard methods for the collection and analysis of data on national mortality have long been available from the World Health Organization (WHO) [1]. There are two separate parts to data collection. The first is the total population census and the second is the recording of deaths in that population. An electronic literature search in July 2005 (using Science Citation, Medline and Embase as databases and cystic fibrosis (CF), mortality, survival and incidence as search terms) showed four national censuses of a CF population for the purpose of calculating disease incidence. They were: one for Sweden for the period 1950-1957 [2], one for the Netherlands for 1961-1965 [3], one for the former Czechoslovakia (now Czech Republic) for 19601967 [4] and one for the UK for 1968-1993 [5-7].

The UK census data, which was collected by the UK Cystic Fibrosis Survey (UKCFS) until 1997, has been linked with subsequent national death registration data, thus attempting to adhere to the WHO criteria for mortality statistics. Death certification data for children in England and Wales showed a striking decline in post-neonatal mortality from CF between 1968 and 2000 and also a steady but less marked decline among 1$5 \mathrm{yr}$ olds [8]. The present report expands this information to include adults and compares data from successive cohorts.
The sources of the data have previously been described in detail elsewhere [5]. Briefly, data were initially obtained by regular surveys sent to all paediatricians and chest physicians in the UK, among others. Later, the number of respondents was reduced by eliminating those who stated that they did not look after CF patients, usually naming colleagues who did. Clinicians who did not respond by the due date were reminded, usually by telephone calls, and each survey was not concluded until the coordinator was satisfied that all the data up to the end of an agreed calendar year had been obtained. This meant that published reports were irregular and not annual, as originally hoped. The UKCFS is unique in its coverage of the CF population, being clinicianbased and not clinic-based, and previous surveys indicated that it included $>95 \%$ of patients. Although there is a wide variety of CF phenotypes, which may to some extent modify clinical presentation and apparent incidence in different populations [9], the common approach to patient management in many countries suggests that, in the absence of other data, the UK mortality data can be taken as illustrative of a general trend.

As a further report in the present authors' series based on UKCFS data, an updated cohort survival data and current survival data for the UK is presented in the current study, including,
AFFILIATIONS

*Dept of Child Health, University of Wales Swansea, Swansea, and "Dept of Mathematical Sciences, University of Bath, Bath, UK.

CORRESPONDENCE

J.A. Dodge

Dept of Child Health

University of Wales Swansea

Singleton Hospital

Sketty Road

Swansea SA2 8QA

UK

Fax: 441291671364

E-mail: j.a.dodge@btinternet.com

Received:

July 312006

Accepted after revision:

November 272006

SUPPORT STATEMENT

The active surveillance was supported by a grant from the Cystic Fibrosis Trust between 1983 and 1997. The death certification data reported were purchased from the relevant authorities in England, Wales, Scotland and Northern Ireland using funds from the University of Bath (Bath, UK) and the University of Wales Swansea (Swansea, UK). № competing interests have been declared. J.A. Dodge initiated the UK Cystic Fibrosis Survey study and followed up unmatched death certificates. P.A. Lewis suggested the present paper and calculated the cohort survival. M. Stanton calculated the current survival and expectation of life. J. Wilsher handled the lefttruncated survival and the population estimate and incidence. All authors contributed to drafting the paper.

STATEMENT OF INTEREST

None declared.

European Respiratory Journal Print ISSN 0903-1936 Online ISSN 1399-3003 
for the first time, life tables with the (tentative) expectation of life for people with CF.

\section{METHODS \\ Data}

Active surveillance of the CF population ran over the period 1982-1997 under the auspices of the (then) British Paediatric Association and the British Thoracic Society, and was funded by the Cystic Fibrosis Trust. The present study was essentially a follow-up of a previous report elaborated in 1997 [6] using the subset of the 1997 data utilised in previous mortality calculations. Personal identification data were removed before passing it to the statisticians. It included all known individuals resident in the UK with a diagnosis of CF who were either born since 1968 or before 1968 and still were alive in 1977. Death certification data up to the end of 1995 were used. It was accepted that ascertainment would be incomplete for the cohorts from 1990 either because some patients had not yet been diagnosed or due to delays inherent to the data collection methods [6]. Strict confidentiality was maintained and the identity of individuals was known only to the corresponding consultant paediatrician or adult physician and the nonmedical coordinator of UKCFS. All data released from UKCFS were aggregated and anonymous.

In the present study, the data held in 1997 were supplemented by data from all death certificates for UK residents from 1996 up to the end of 2003 in which CF or any of its synonyms were mentioned (International Classification of Diseases (ICD)-9 codes 2270, 7770 and 7484; and ICD-10 codes E84.0, E84.1, E84.8 and E84.9). Prior to version 6, introduced in 1968, ICD did not separate CF from other diseases of the pancreas, so death certification data was unreliable. The legal definition of the minimum term for a stillbirth/neonatal death changed in 1996 from 28 to 24 weeks. The legal definition of a neonatal death was used, which was relevant for one case.

Death certification data were linked to the UKCFS database only using date of birth and sex. For the purposes of mortality calculation it is only necessary to record one of any duplicate matches. Where there was no match for death certificate data, checks were made by contacting the certifying physician and the data were corrected where necessary. Dead patients not previously known to UKCFS were included as new cases, unless positive evidence was found of their ineligibility, such as foreign residents who died while temporarily in the UK, e.g. visiting relatives or requesting a transplant.

The age/sex structure of the 2003 mid-year CF population of $\geqslant 12$ yrs of age is known directly. To estimate the numbers in the
2003 population of $<12$ yrs of age, the total UK live births for each year were found first. For these data, the CF births could be estimated using an incidence of one in 2,381 (as reported in the present study). The survival of each of these cohorts up to 2003 was presumed to follow the survival of the 1992-1994 cohort, which leads directly to the estimated numbers surviving.

Incidence was only calculated for the period 1968-1987 due to concerns regarding later under-ascertainment.

Cohort survival was calculated using the life table method and current survival was calculated by applying the age/sexspecific mortality rates for $1 \mathrm{yr}$ successively to a hypothetical birth cohort [10]. For the left-truncated survival, the total UK live births were known for each period and therefore the total $\mathrm{CF}$ births could be inferred using the incidence. The survey data gave the size of the CF population that survived to a specific age; the proportion of those who survived follows. The remaining years survival was calculated using the life table method. The data for the two sexes were pooled due to small numbers in the older age groups.

\section{RESULTS \\ Deaths}

There were 1,066 deaths from 1996-2003, at a mean (range) of 133 (120-148) deaths $\cdot \mathrm{yr}^{-1}$. In the cohorts born since 1968 there were 872 deaths, 432 (49\%) males in the same time period.

\section{Population}

Table 1 gives an estimate of the size of the mid-2003 UK CF population. The size of the age group 0-9 yrs was estimated based on extrapolations from the UK live births and the age group $\geqslant 10$ yrs from data held. These data form the denominator in the current survival calculations. The 1992 population is given as 6,740 .

\section{Incidence}

The extra cases found through the present study have led to a $1.4 \%$ increase in the previous incidence estimate for 1968-1987 from one in 2,416 [6] to one in 2,381 live births $(6,474$ / $\left.15.4 \times 10^{6}\right)$.

\section{Survival}

The survival of 3-yr cohorts of male and female patients born in the period 1968-1994 is given in figures $1 \mathrm{a}$ and $\mathrm{b}$, with corresponding hazard rates in table 2. The updated [11] truncated survival in 3-yr cohorts for CF patients born in the period 1947-1970 is given in figure 2. The current survival for

TABLE 1 Mid-2003 cystic fibrosis population by age and sex

\begin{tabular}{|c|c|c|c|c|c|c|c|c|c|c|c|c|}
\hline Male & 140 & 550 & 700 & 725 & 152 & 143 & 992 & 672 & 318 & 40 & 7 & 4439 \\
\hline Total & 280 & 1100 & 1400 & 1359 & 280 & 283 & 1794 & 1151 & 539 & 79 & 19 & 8284 \\
\hline$\%$ & 3.4 & 13.3 & 16.9 & 16.4 & 3.4 & 3.4 & 21.7 & 13.9 & 6.5 & 1.0 & 0.2 & 100.0 \\
\hline
\end{tabular}

The size of the 0-9-yrs-old-age group was estimated from population births and survival data. The size of the age groups of $\geqslant 10$ yrs of age was based on data held by the UK Cystic Fibrosis Survey. 

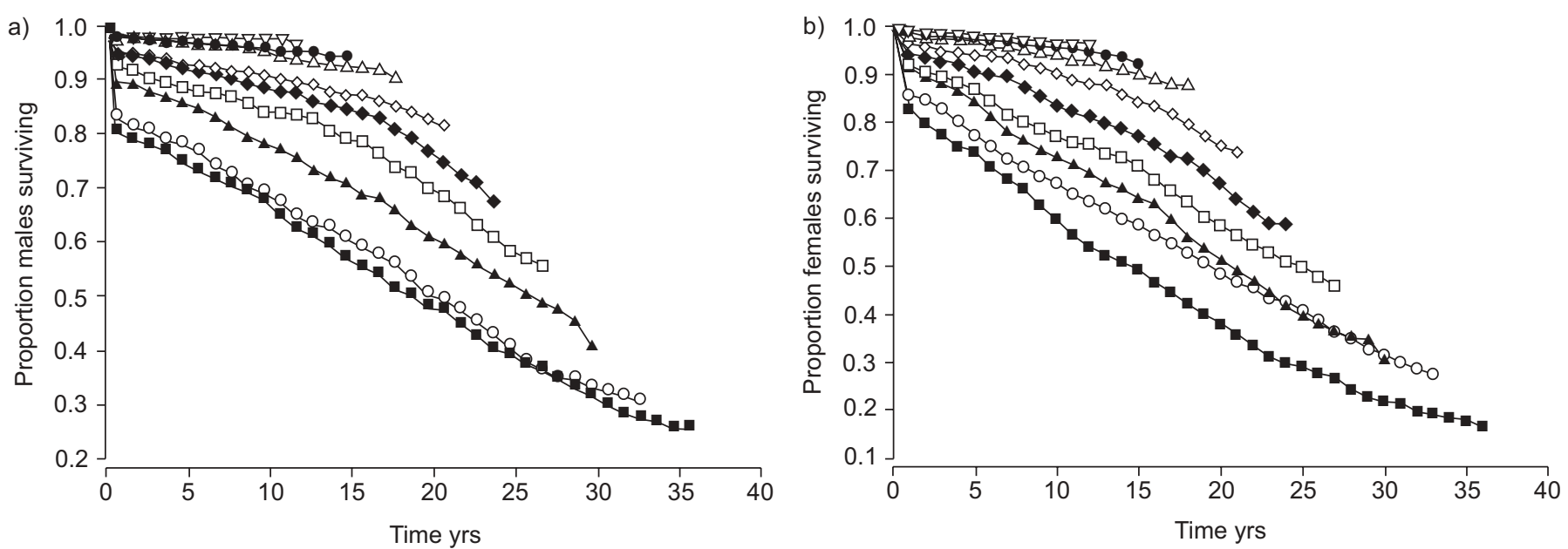

FIGURE 1. UK cystic fibrosis population. Proportion of a) males and b) females of each 3-yr cohort surviving until 2003.

1968-1970; O: 1971-1973;

1974-1976

口: 1977-1979;

: 1980-1982; $\diamond:$ 1983-1985; $\triangle$ : 1986-1988;

• : 1989-1991; $\nabla:$ : 1992-1994.

males and females is given in figure 3. An abridged expectation of life table is given in table 3 .

\section{DISCUSSION}

The results presented in the current paper show that the continued improvement in survival anticipated from previous results is occurring. The median expectation of life for the $\mathrm{CF}$ population for 2000-2003 estimated from the current survival calculations averages $\sim 40$ yrs (fig. 3 ). These calculations assume that the current age-specific mortality rates will continue. However, their continued improvement, as reflected in table 2, suggests that an estimated median survival of $50 \mathrm{yrs}$ [12] for the birth cohort of the year 2000 remains likely.

The small number of death certificates of individuals born prior to 1996 who were not known to UKCFS and previous experience of the active surveillance in uncovering deaths not reported via the certification authority, confirm that these data should not be overinterpreted. During the active surveillance period, great care was taken to avoid duplicating cases. The subsequent merging of post-1996 death data is not entirely satisfactory, since patients in the UKCFS database were identified only by sex and date of birth. It is possible that some existing cases marked as "deceased" were new cases and some deceased marked as "new" were already in the data set, perhaps under another name. However, the follow-up enquiries and discussion with the relevant physician about new deceased cases should have almost eliminated this risk. The numbers are certainly small, may partially balance out and do not contribute any important errors to these results.

The effects of the genuinely new cases previously unknown to UKCFS are to increase previous estimates of the population size and to slightly improve the historical survival. The effects may be seen by reference to previous work: for example, the 1992 population is now given as 6,740 compared with a previous count of 6,499 [6] and an earlier projection of 6,000 [7]. After excluding deaths among individuals of other nationalities, newly or temporarily resident in the UK at the time of death, and misdiagnoses, the remaining cases new to UKCFS suggest that there are still patients with CF born before 1995 who were not identified by the survey. Nearly all of the new cases were late diagnoses, either presenting with an atypical history or found by family case studies. Whatever the reasons for being unknown to UKCFS, the clear implication is that there are still some unreported cases that could affect these data. In terms of the survival data, a proportion, albeit small, of the cohort-by-cohort increase can be attributed to a greater ascertainment of late-diagnosed, milder cases. All patients included here have CF. If there is to be any true appreciation of trends in survival, it is important that criteria for diagnosis are consistent and that patients with CF transmembrane conductance regulator-related disorders, such as isolated obstructive azoospermia, which do not meet the diagnostic criteria of CF and are not known to have an impact on life expectancy, are excluded from consideration.

The previously noted pattern of linear descent of the survival curves continues, following the addition of the further data (fig. 1). The historical better early survival of males with CF is much less apparent, although the survival by sex differs markedly for cohorts born before 1987. It is still not clear whether this is a cohort effect or whether adult females inherently have a worse survival than males. As the age at which the survival curves separate has increased recently, reduction of the adult sex gap may continue.

The truncated survival curves shown in figure 2 (truncated as no data was available for the older cohorts to deduce their shape before 1968) show that a steadily increasing proportion is surviving into their forties and beyond. The characteristics, evolving problems and outlook for these long-surviving patients are the subject of ongoing international studies $[13,14]$.

Specific survival data that may help in informing patients and their carers are given in table 3. Death from CF in the first decade of life is now rare [15]. This is presumably related to steadily improving clinical management. Earlier cohorts show a striking reduction in deaths in the first year of life, which was associated with the (now almost universal) survival of infants with meconium ileus resulting from improved neonatal management $[6,7]$ (figs. 1 and 2). The post-infancy rate of attrition depicted in 
TABLE 2 Cohort survival of the UK cystic fibrosis (CF) population by sex based on data held to the end of 2003

\begin{tabular}{|c|c|c|c|c|c|c|c|c|c|}
\hline & \multicolumn{9}{|c|}{ Year of birth } \\
\hline & $1968-1970$ & $1971-1973$ & 1974-1976 & 1977-1979 & 1980-1982 & 1983-1985 & $1986-1988$ & 1989-1991 & 1992-1994 \\
\hline \multicolumn{10}{|l|}{ Males } \\
\hline CF births $n$ & 592 & 545 & 482 & 469 & 534 & 472 & 467 & 482 & 396 \\
\hline \multicolumn{10}{|l|}{ Age yrs } \\
\hline $0-<1$ & 213.1 (114) & $182.2(91)$ & $111.7(51)$ & 77.5 (35) & $47.9(25)$ & $54.5(25)$ & $23.8(11)$ & $18.8(9)$ & $22.9(9)$ \\
\hline $1-<5$ & $18.4(34)$ & $15.3(27)$ & 10.7 (18) & $11.2(19)$ & $8.5(17)$ & $5.7(10)$ & $2.8(5)$ & $3.2(6)$ & $0.6(1)$ \\
\hline $5-<10$ & $19.4(41)$ & $23.8(48)$ & $18.8(37)$ & 10.9 (22) & $8.3(20)$ & $4.6(10)$ & $3.1(7)$ & $2.6(6)$ & $0(0)$ \\
\hline $10-<15$ & $33.9(63)$ & $25.8(46)$ & $19.5(35)$ & $11.5(22)$ & $9.1(21)$ & $7.6(16)$ & $5.9(13)$ & $4.3^{\# \#}(5)$ & $1.5^{\#}(1)$ \\
\hline $15-<20$ & $33.8(53)$ & $36.7(56)$ & $29.6(47)$ & $24.6(43)$ & 19.5 (42) & $12.8^{\#}(17)$ & $2.7^{\#}(3)$ & & \\
\hline $20-<25$ & $39.8(52)$ & $42.3(53)$ & $30.0(41)$ & $41.7^{\#}(52)$ & $26.4^{\#}(27)$ & & & & \\
\hline $25-<30$ & $42.4(45)$ & $45.3(40)$ & $50.6^{\#}(32)$ & & & & & & \\
\hline $30-<35$ & $54.1^{\#}(31)$ & $15.3^{\#}(7)$ & & & & & & & \\
\hline \multicolumn{10}{|l|}{ Females } \\
\hline CF births $n$ & 530 & 514 & 434 & 419 & 439 & 461 & 446 & 418 & 363 \\
\hline \multicolumn{10}{|l|}{ Age yrs } \\
\hline $0-<1$ & $190.0(92)$ & $155.1(74)$ & $901.6(38)$ & $87.1(35)$ & $61.0(26)$ & $39.8(18)$ & $24.9(11)$ & $14.4(6)$ & $13.9(5)$ \\
\hline $1-<5$ & $28.3(47)$ & $25.6(43)$ & $20.3(31)$ & $13.3(20)$ & $9.9(16)$ & $5.7(10)$ & 1.7 (3) & $3.0(5)$ & $2.1(3)$ \\
\hline $5-<10$ & $41.8(74)$ & $27.4(51)$ & $29.4(50)$ & $23.8(41)$ & $16.3(31)$ & $8.0(17)$ & $6.5(1)$ & $3.5(7)$ & $5.1(6)$ \\
\hline $10-<15$ & $38.7(56)$ & $27.8(45)$ & $24.9(37)$ & $16.7(26)$ & $16.5(29)$ & $13.9(28)$ & $7.8(1)$ & $6.0^{\#}(6)$ & $0^{\#}(0)$ \\
\hline $15-<20$ & $51.9(60)$ & $38.6(53)$ & $44.5(56)$ & $39.1(53)$ & $27.3(43)$ & $28.7^{\#}(36)$ & $6.9^{\#}(7)$ & & \\
\hline $20-<25$ & $54.2(48)$ & $34.1(40)$ & $51.9(51)$ & $37.2(35)$ & $38.0^{\#}(28)$ & & & & \\
\hline $25-<30$ & $55.0(37)$ & $50.6(47)$ & $27.9^{\#}(22)$ & & & & & & \\
\hline $30-<35$ & $53.5^{\#}(19)$ & $27.2^{\#}(11)$ & & & & & & & \\
\hline
\end{tabular}

Data presented as hazard rate, which is the instantaneous mortality rate, per thousand, with the number of deaths in parentheses, unless otherwise stated. Censored data with $<100$ life yrs of observation and $<10$ deaths have been omitted. The standard error of the hazard rate for all the $0-1$-yr-old age groups is $\sim 10$ per thousand, for the $1-5$-yrs-old group it is approximately four per thousand rising to six per thousand for the 30-35-yrs-old group. \# : data subject to censoring.

the cohort survival curves has consistently flattened out, suggesting that with that exception, no single new therapeutic intervention can be identified as a major reason for the improved survival. In 1968 in England and Wales, there were 118 deaths

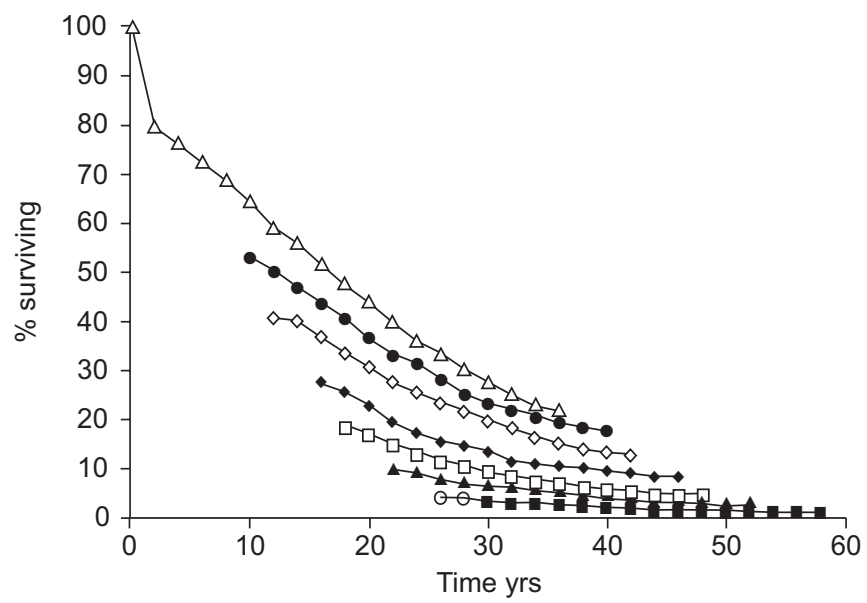

FIGURE 2. Left-truncated survival curves up to 2003 of the UK cystic fibrosis population born in the period 1947-1970 in 3-yr cohorts. $\mathbf{~ : ~ 1 9 4 7 - 1 9 4 9 ; ~ O : ~ 1 9 5 0 - ~}$ 1052; \: 1953-1955; $\square: 1956-1958 ; \diamond: 1959-1961 ; \diamond: 1962-1964 ; \bullet: 1965-$ 1967; $\triangle:$ : 1968-1970 attributable to $\mathrm{CF}$ in children aged 28 days to $16 \mathrm{yrs}$ old, compared with 75 in 1985 and 15 in 2000 [8].

This makes the cohort effect the major determinant of survival and much stronger than sex. Occasionally, CF patients die from unrelated causes, such as road traffic accidents. One such

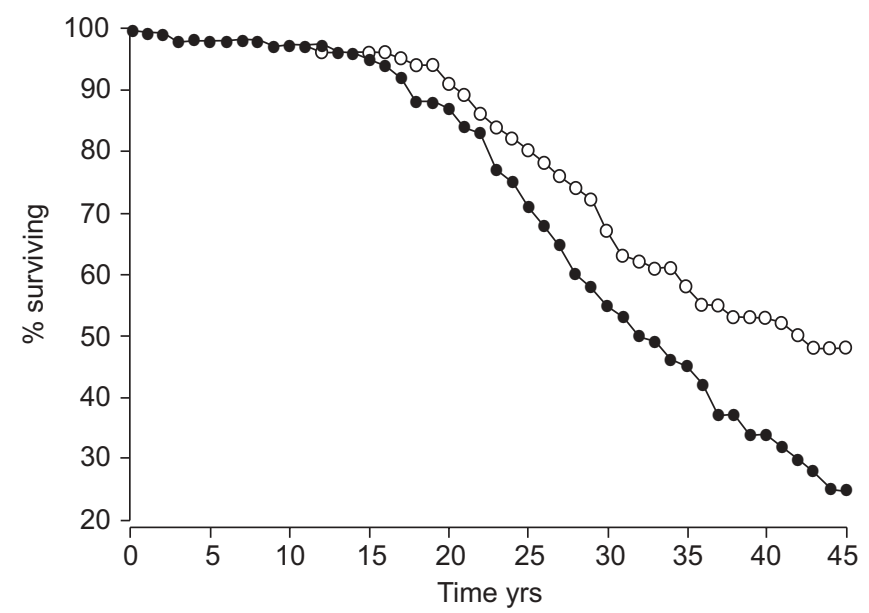

FIGURE 3. Current survival UK cystic fibrosis population by sex, 2003. 0 : males; $\bullet$ females. 


\begin{tabular}{lcc} 
TABLE 3 & Abridged expectation of life by sex: 2003 \\
Age yrs & \multicolumn{2}{c}{ Expected mean age of death yrs } \\
\cline { 2 - 3 } & Males & Females \\
\hline $\mathbf{0}$ & 42.62 & 36.89 \\
$\mathbf{1}$ & 43.12 & 37.33 \\
$\mathbf{2}$ & 43.12 & 37.33 \\
$\mathbf{3}$ & 43.25 & 37.44 \\
$\mathbf{4}$ & 43.25 & 37.44 \\
$\mathbf{5}$ & 43.38 & 37.55 \\
$\mathbf{1 0}$ & 43.63 & 37.76 \\
$\mathbf{1 5}$ & 44.18 & 38.39 \\
$\mathbf{2 0}$ & 45.41 & 40.43 \\
$\mathbf{2 5}$ & 48.66 & 44.34 \\
$\mathbf{3 0}$ & 52.67 & 49.40 \\
\hline
\end{tabular}

instance during the period covered here is known but, unless CF was mentioned on the death certificate, this would not be the case. However, such deaths would be few, would have the same background mortality as the general age-matched population and would not obscure the observation that survival of people with CF continues to improve.

The data for the expectation of life may lead to more realistic life insurance being made available to people with CF phenotype. Conversely, these results should lead to a lower benefit being available for an impaired life annuity.

\section{Comparisons with other studies}

A proper comparison with the results from other published data sets presents serious technical and methodological problems. As these data sets do not conform to the WHO standard of a total defined population, a noncohort analysis is required. The results from these analyses are frequently misinterpreted, as comparisons are made using relative risks (or odds ratios) with insufficient explanation [16]. Other examples of problems in interpreting $\mathrm{CF}$ survival data are available [17]. However, survival data from large national registries which, unlike UKCFS, are based on information only from specialist clinics and therefore have a selection bias, show broadly comparable and gratifying trends [18, 19].

\section{Conclusions}

1) The total population study shows that survival with cystic fibrosis continues to improve in the UK and, by implication, in many other countries too.

2) Continued growth of the adult cystic fibrosis population by $\sim 145$ patients per annum has national and local implications for healthcare provision and training of staff to manage adult cystic fibrosis clinics. The annual population increase would approximate to the size of a moderately large adult clinic.

3) The expectation of life data should be of value to life insurance companies in calculating risks and returns on life annuities for people with cystic fibrosis.

4) Previously published predictions of a mean survival of $>50$ yrs of age, for infants with cystic fibrosis recently born in the UK, continue to look realistic, even in the absence of proven effective therapy aimed at correcting the basic cystic fibrosis defect.

\section{ACKNOWLEDGEMENTS}

These data were collected over many years with the generous assistance of over 1,500 carers of people with cystic fibrosis.

\section{REFERENCES}

1 Manual of mortality analysis. Geneva, World Health Organization (Division of Health Statistics), 1977; pp. 2-3.

2 Brunecky Z. The incidence and genetics of cystic fibrosis. J Med Genet 1972; 9: 33-37.

3 Ten Kate LP. Cystic fibrosis in the Netherlands. Int $J$ Epidemiol 1977; 6: 23-34.

4 Selander P. The frequency of cystic fibrosis of the pancreas in Sweden. Acta Paediatr 1962; 51: 65-67.

5 Cystic fibrosis in the United Kingdom 1977-85, an improving picture. British Paediatric Association Working Party on Cystic Fibrosis. BMJ 1988; 297: 1599-1602.

6 Dodge JA, Morison S, Lewis PA, et al. Incidence, population, and survival of cystic fibrosis in the UK, 1968-95. Arch Dis Child 1997; 77: 493-496.

7 Dodge JA, Morison S, Lewis PA, et al. Cystic fibrosis in the United Kingdom, 1968-1988: incidence, population and survival. Paediatr Perinat Epidemiol 1993; 7: 157-166.

8 Panickar JR, Dodd SR, Smyth RL, Couriel JM. Trends in deaths from respiratory illness in children in England and Wales from 1968-2000. Thorax 2005; 60: 1035-1038.

9 The molecular genetic epidemiology of cystic fibrosis Report of a joint meeting of WHO/ECFTN/ICF(M)A/ ECFS. Geneva, WHO Human Genetics Programme, 2004; p. 24. Available at http://whqlibdoc.who.int/hq/2004/ WHO_HGN_CF_WG_04.02.pdf.

10 Armitage P, Berry G. Statistical methods in medical research. Oxford, Blackwell Science Ltd, 1994; p. 470.

11 Lewis PA, Morison S, Dodge JA, et al. Survival estimates for adults with cystic fibrosis born in the United Kingdom between 1947 and 1967. Thorax 1999; 54: 420-422.

12 Jannot A-S. Examples of statistical analysis of epidemiological data sets. MPhil Dissertation. University of Bath, Bath, 2000.

13 Dodge JA, Milla CE, Warwick WJ. A study of cystic fibrosis (CF) associated diseases of aging. J Cyst Fibros 2004; 3: S122.

14 University of Minnesota. Aging with Cystic Fibrosis: Organization of an International Project. http://www.med. umn.edu/agingcf/. Date last updated: November 5, 2004.

15 Dodge JA, Lewis PA. Cystic fibrosis is no longer an important cause of childhood death in the United Kingdom. Arch Dis Child 2005; 90: 547.

16 Bradford Hill A. Further fallacies and difficulties. Proportional rates. In: A short textbook of medical statistics. Kent, Hodder and Stoughton, 1977; p. 266.

17 Lewis PA. Inferences for health provision from survival data in cystic fibrosis. Arch Dis Child 1998; 79: 297-299.

18 Cystic Fibrosis Foundation, Patient Registry. Annual Report 2004. Bethesda, CFF, 2004.

19 McCormick J, Sims EJ, Green MW, Mehta G, Culross F, Mehta A. Comparative analysis of Cystic Fibrosis Registry data from the UK with USA, France and Australasia. J Cyst Fibros 2005; 4: 115-122. 